\title{
INTEGRACIÓN DE CONTROL BIOLÓGICO Y DE PRODUCTOS ALTERNATIVOS CONTRA Tetranychus urticae (ACARI: TETRANYCHIDAE)
}

\section{INTEGRATION OF BIOLOGICAL CONTROL AND ALTERNATIVE PRODUCTS AGAINST Tetranychus urticae (ACARI: TETRANYCHIDAE)}

\author{
Alberto Soto ${ }^{1}$, Hamilton G. Oliveira ${ }^{2}$, Angelo Pallini ${ }^{3}$ \\ ${ }^{1}$ I.A., M.Sc., Ph.D. Profesor Universidad de Caldas. Calle 65 No 26-10 Manizales, Caldas, Colombia; alberto.soto@ucaldas.edu.co \\ ${ }^{2}$ Ph.D. Investigador Centro Nacional de Investigaciones en Palma, CENIPALMA, Villavicencio, Colombia; hgomes@cenipalma.org \\ ${ }^{3}$ Ph.D. Profesor Universidade Federal de Viçosa. CEP 36570-000, Viçosa, MG, Brasil; pallini@ufv.br
}

Rev. U.D.C.A Act. E Div. Cient. 14(1): 23 - 29, 2011

RESUMEN

Tetranychus urticae es una importante plaga asociada al cultivo de fresa debido a las altas densidades poblacionales alcanzadas y a los daños ocasionados a las plantas. Los productores utilizan acaricidas en grandes volúmenes sin cumplir los periodos de carencia, ocasionando residuos tóxicos en los frutos, desarrollo de poblaciones resistentes a los productos, destrucción de organismos benéficos, intoxicación de mamíferos y contaminación del medio ambiente. Por lo tanto, es necesario generar otras alternativas de manejo de este ácaro plaga. El objetivo de este trabajo fue evaluar, mediante el análisis conjunto de la concentración letal y subletal, la acción tóxica de productos comerciales a base de nim sobre los ácaros T. urticae y Phytoseiulus macropilis. Fueron controladas poblaciones de T. urticae en plantas de fresa cuando se emplearon concentraciones correspondientes a $\mathrm{CL}_{95}$ de los productos alternativos a base de nim, y también cuando se utilizaron concentraciones inferiores. La aplicación de los productos a base de nim, Neem Pro, Organic Neem y Natuneem en la $\mathrm{CL}_{95}$ y cuando la tasa instantánea de crecimiento poblacional $\mathrm{ri}=0,1$ para el predador representan una alternativa viable para el control del ácaro fitófago T. urticae.

Palabras clave: Acaro rayado, Phytoseiulus macropilis, Azadirachta indica, ácaros fitófagos, fresa.
SUMMARY

Tetranychus urticae is an important pest associated with strawberry, due to its high densities and plant damage. Farmers use acaricides in large volumes without complying with the window between the last application and harvest, resulting on toxic residues in fruits, as well as pest populations resistant to pesticides, decrement of beneficial organisms, mammalian toxicity and environmental pollution. Therefore, it is necessary to generate new alternatives for the control of this mite. The aim of this work was to evaluate the toxic effect of commercial neem-based products on the mites T. urticae and Phytoseiulus macropilis, through a combined analysis using both, lethal and sublethal concentrations. Tetranychus urticae populations were controlled in strawberry plants when neem was used at or below $\mathrm{LC}_{95}$ concentrations. The application of the neem-based products, Neem Pro, Organic Neem and Natuneem at $\mathrm{CL}_{95}$, and when the instantaneous predator's rate of population increase $r i=0.1$, represents a viable alternative to be considered for the control of the phytophagous mite T. urticae.

Key words: Striped mite, Phytoseiulus macropilis, Azadirachta indica, phytophagous mites, strawberry.

\section{INTRODUCCIÓN}

Tetranychus urticae Koch (Acari: Tetranychidae) es considerada una de las principales plagas asociadas al 
cultivo de fresa. En Brasil, T. urticae ocasiona pérdidas hasta del $80 \%$ de la producción, debido, principalmente, a su alto potencial reproductivo (Chiavegato $\mathcal{E}$ Mischan, 1981). Este hecho ha llevado a los productores a emplear acaricidas en grandes volúmenes, ocasionando altos niveles de residuos tóxicos en los frutos, desarrollo de poblaciones resistentes a los productos, intoxicación de mamíferos y destrucción de organismos benéficos (Hernández E Vendramim, 1996; Sato et al. 2000; Venzon et al. 2008). La utilización de compuestos naturales extraídos de plantas y la liberación del ácaro predador Phytoseiulus macropilis (Banks) (Acari: Phytoseiidae), el cual, presenta gran eficiencia predatoria, puede contribuir con el éxito de programas de manejo integrado de T. urticae (García E Chiavegato, 1997; Potenza et al. 1999a,b; Gonçalves et al. 2001; Brito et al. 2006; Oliveira et al. 2007).

El nim Azadirachta indica A. Juss, cuyo principal compuesto activo es la azadirachtina, presenta elevada acción insecticida y acaricida, bajísima toxicidad al hombre y a animales domésticos (Rembold, 1989; Castagnoli et al. 2000; ElGengaihi et al. 2000; Mourão et al. 2004). El producto se ha mostrado bastante promisorio en el manejo integrado de ácaros fitófagos, causando mortalidad, reducción de la fecundidad, deterrencia, inviabilidad de formas inmaduras y repelencia (Dimetry et al. 1993; Mansour et al. 1993; Spollen E Isman, 1996; Momen et al. 1997; Schmutterer, 1997; Castiglioni et al. 2002; Brito et al. 2006; Soto et al. 2010). Además, la planta posee multiplicidad de compuestos, como la solanina, la azadiradiona y la azadirachtina, dificultando el surgimiento de poblaciones resistentes (Viegas Jr., 2003).

Individuos que sobreviven a la exposición a pesticidas pueden sufrir efectos subletales (Venzon et al. 2008). Estos efectos pueden ser manifestados a través de la reducción en el período de vida, disminución de fertilidad, reducción de la fecundidad, cambios en la razón sexual y en el comportamiento de alimentación y oviposición (Vinson, 1974; Stark et al. 1992; Stark E Rangus, 1994).

Por lo tanto, la verificación solamente de efectos letales sobre los organismos podrá no detectar importantes efectos sobre los herbívoros y los enemigos naturales. Los parámetros que definen el efecto letal o subletal son las concentraciones empleadas de los productos (Venzon et al. 2008).

El objetivo de este trabajo fue evaluar los efectos letales y subletales de productos derivados de nim sobre los Ácaros $T$. urticae y $P$. macropilis.

\section{MATERIALES Y MÉTODOS}

El trabajo, se realizó en el laboratorio de Acarología del Departamento de Biología Animal, de la Universidade
Federal Viçosa, Minas Gerais, Brazil. Fueron estudiados los efectos letales y subletales de los productos comerciales a base de nim, con la concentración de azadirachtina: Neem Pro (10mg/L de azadirachtina), Natuneem (1,5mg) y Organic Neem (3,3mg) sobre T. urticae y $P$. macropilis.

Para determinar la selectividad de compuestos a enemigos naturales han sido aplicados, en gran medida, los índices de toxicidad diferencial, normalmente, determinados a través de test de toxicidad aguda, con el uso de las concentraciones letales (CL) estimadas (Sato et al. 2002; Stark \& Banks, 2003). A pesar de ser una medida importante, la CL es una medida incompleta de los efectos de productos químicos sobre poblaciones, pues analiza solamente la mortalidad, como parámetro de toxicidad.

El análisis de la tasa de crecimiento poblacional ha sido recomendado en conjunto con el cálculo de la CL, para el estudio de efectos tóxicos a artrópodos en laboratorio, ya que combinan efectos letales y subletales (Walthall $\mathcal{E}$ Stark, 1997). La tasa instantánea de crecimiento poblacional (ri) es una medida directa de crecimiento poblacional en determinado período de tiempo pudiendo ser adoptada para predecir el crecimiento poblacional de artrópodos (Walthall E Stark, 1997)

Fueron estimadas las concentraciones letales (CL) de los productos a base de nim para hembras adultas de $T$. urticae y $P$. macropilis. Las concentraciones evaluadas fueron escogidas a través de bioensayos iniciales y se situaron entre el límite inferior, donde el producto no causó mortalidad y el límite superior de respuesta, donde generó $100 \%$ de mortalidad para cada especie.

Los bioensayos de concentración-respuesta fueron realizados utilizándose hembras de los ácaros $T$. urticae y $P$. macropilis, al inicio de la fase reproductiva. Los productos fueron pulverizados en discos $(\varnothing=3,0 \mathrm{~cm})$ de hojas de fresa, acondicionados en caja de Petri, a través de torre de Potter, Burkard, Rickmansworth, UK (Potter, 1952), sobre la presión de $5 \mathrm{lb} / \mathrm{pul}^{2}$ y con la aplicación de un volumen igual a 2,5mL por dosis, lo que corresponde a un depósito de 1,70 $\pm 0,07 \mathrm{mg} / \mathrm{cm}^{2}$, sobre la superficie tratada. Esta cantidad aplicada está de acuerdo con lo recomendado por la IOBC/ WPRS (Internacional Organization for Biological Control of Noxious Animals and Plants/ West Paleartic Regional Section) (Overmeer E Van Zon, 1982) y representa lo que ocurre en situación de campo (Reis et al. 1998). Para la fijación del disco en la placa, se preparó una solución de carragenina (30g/300mL de agua), siendo que el disco fue colocado antes que la solución se solidificara.

Los discos asperjados con los productos y con agua (testigo) fueron expuestos al ambiente por una hora, para el secado del producto; posteriormente, ocho hembras de los ácaros $T$. urticae fueron colocadas sobre cada disco. Para P. macropilis 
(igualmente a lo realizado para $T$. urticae), los discos de hojas de fresa fueron pulverizados, secos e infestados con T. urticae; después, los discos recibieron ocho hembras del ácaro predador $P$. macropilis; cada concentración del producto fue repetida cinco veces. Los discos de hojas tratados fueron mantenidos en cámara climatizada (25 \pm $2^{\circ} \mathrm{C}, 60 \pm 10 \%$ HR y $13 \mathrm{~h}$ de luz); la mortalidad fue evaluada 24 h después a la aplicación de los productos y las curvas de concentración-mortalidad fueron estimadas por el análisis de Probit (Finney, 1971).

La evaluación de la acción subletal de los productos alternativos a base de nim sobre el ácaro fitófago $T$. urticae y el predador $P$. macropilis fue aplicada utilizando la misma metodología citada para la evaluación de la acción letal de los productos; el tiempo de evaluación fue de cinco días.

Para cada concentración de cada producto fueron realizadas cinco repeticiones, donde cada una fue representada por un disco con ocho hembras de T. urticae. Los ácaros fueron mantenidos en cámaras climatizadas sobre las mismas condiciones descritas en los experimentos de toxicidad letal. La evaluación del efecto subletal, se realizó a través de la estimativa de la tasa instantánea de crecimiento $\left(\mathrm{r}_{\mathrm{i}}\right)$, por la siguiente fórmula (Stark E Banks, 2003):

$$
r_{i}=\ln \left(N_{f} / N_{0}\right) / \Delta t
$$

Donde $\mathrm{N}_{\mathrm{f}}$ es el número final de individuos, $\mathrm{N}_{0}$ es el número inicial de individuos, $\Delta$ t es la variación de tiempo (duración del experimento cinco días). El valor positivo de $r_{i}$, significa que la población está en crecimiento; $r_{i}=0$, indica que la población está estable, mientras que un valor negativo de $r_{i}$ señala que la población está en descenso y en vías de extinción. El análisis de regresión, se aprovechó para la evaluación de $r_{i}$ en función de las concentraciones utilizadas.

\section{RESULTADOS Y DISCUSIÓN}

\section{Toxicidad letal}

Para el Neem Pro fueron obtenidas $\mathrm{Cl}_{50}$ de 0,06 y 0,07 $\mathrm{mg} / \mathrm{L}$ de azadirachtina para $T$. urticae y $P$. macropilis, respectivamente. Las $\mathrm{Cl}_{95}$ fueron de 0,27 y 0,22 mg i.a/L para T. urticae y $P$. macropilis, respectivamente (Tablas 1 y 2).

El ácaro predador $P$. macropilis presentó mayor selectividad al producto Neem Pro (1,2 veces más resistente que $T$. urticae), lo que indica que en la concentración de 0,06 mg i.a/L de este producto comercial, se controla el ácaro fitófago $T$. urticae y no se afecta el predador. La toxicidad o selectividad a los ácaros puede variar, sobre todo, en función del solvente utilizado en la extracción, parte de la planta procesada y concentración aplicada (Mansour E Ascher, 1983; Mansour et al. 1987; Castiglioni et al. 2002; Mourão et al. 2004) (Tablas 1 y 2).

Para Organic Neem fueron obtenidas $\mathrm{Cl}_{50}$ de 0,29 y 0,17mg/L de azadirachtina para $T$. urticae y $P$. macropilis, respectivamente. Las $\mathrm{Cl}_{95}$ fueron de 0,82 y 0,70mg i.a/L para T. urticae y $P$. macropilis, respectivamente (Tablas 1 y 2 ).

Respecto a Natuneem, las $\mathrm{Cl}_{50}$ fueron de 0,95 y $0,41 \mathrm{mg} / \mathrm{L}$ de azadirachtina para T. urticae y $P$. macropilis, respectivamente. Las $\mathrm{Cl}_{95}$ fueron de 2,88 y 1,49mgi.a/L del producto comercial para T. urticae y $P$. macropilis, respectivamente (Tablas $1 \mathrm{y}$ 2).

Fueron controladas poblaciones de T. urticae en plantas de fresa cuando se aplicaron concentraciones correspondientes a $\mathrm{CL}_{95}$ de los productos alternativos a base de nim y también cuando se utilizaron concentraciones inferiores. En estas concentraciones, la tasa de crecimiento poblacional de $T$. urticae es igual a cero.

Tabla 1. Toxicidad de los productos a base de nim al ácaro fitófago T. urticae.

\begin{tabular}{|l|l|l|c|c|c|c|}
\hline \multicolumn{1}{|c|}{ Producto } & \multicolumn{1}{|c|}{$\mathrm{N}^{1}$} & $\begin{array}{c}\text { Coeficiente } \\
\text { Angular } \pm \mathrm{EP}^{2}\end{array}$ & $\begin{array}{c}\mathrm{Cl}_{50}{ }^{3} \\
\text { (I.C. a 95\%) }\end{array}$ & $\begin{array}{c}\mathrm{Cl}_{95}{ }^{4} \\
\text { (I.C. a 95\%) }\end{array}$ & \multicolumn{1}{|c|}{$X^{2}$} & \multicolumn{1}{c|}{$P$} \\
\hline Neem Pro & 280 & $0,19 \pm 0,01$ & $0,06(0,05-0,07)$ & $0,27(0,20-0,43)$ & 0,33 & 0,86 \\
\hline Organic Neem & 280 & $0,12 \pm 0,23$ & $0,29(0,26-0,32)$ & $0,82(0,69-1,04)$ & 0,38 & 0,79 \\
\hline Natuneem & 280 & $0,34 \pm 0,10$ & $0,95(0,29-1,08)$ & $2,88(1,47-3,79)$ & 0,62 & 0,75 \\
\hline
\end{tabular}

${ }^{1}$ Número de ácaros evaluados

${ }^{2}$ Coeficiente angular y error patrón de la media

${ }^{3}$ Concentración letal media e intervalo de confianza al 95\%

${ }^{4}$ Concentración letal que causa $95 \%$ de mortalidad e intervalo de confianza al 95\% 
Tabla 2. Toxicidad de los productos a base de nim al ácaro predador P. macropilis.

\begin{tabular}{|l|c|c|c|c|c|c|}
\hline \multicolumn{1}{|c|}{ Producto } & $\mathrm{N}^{1}$ & $\begin{array}{c}\text { Coeficiente } \\
\text { Angular } \pm \mathrm{EP}^{2}\end{array}$ & $\begin{array}{c}\mathrm{Cl}_{50}{ }^{3} \\
\text { (I.C. a 95\%) }\end{array}$ & $\begin{array}{c}\mathrm{Cl}_{95}{ }^{4} \\
\text { (I.C. a 95\%) }\end{array}$ & $X^{2}$ & $P$ \\
\hline Neem Pro & 185 & $0,61 \pm 0,10$ & $0,07(0,06-0,08)$ & $0,22(0,19-0,29)$ & 0,79 & 0,99 \\
\hline Organic Neem & 220 & $0,65 \pm 0,14$ & $0,17(0,14-0,21)$ & $0,70(0,60-1,05)$ & 0,68 & 0,92 \\
\hline Natuneem & 330 & $0,69 \pm 0,07$ & $0,41(0,36-0,47)$ & $1,49(1,23-1,93)$ & 0,35 & 0,89 \\
\hline
\end{tabular}

${ }^{1}$ Número de ácaros evaluados

${ }^{2}$ Coeficiente angular y error patrón de la media

${ }^{3}$ Concentración letal media e intervalo de confianza al 95\%

${ }^{4}$ Concentración letal que causa $95 \%$ de mortalidad e intervalo de confianza al 95\%

\section{Efecto subletal}

Para Neem Pro, la tasa instantánea de crecimiento poblacional fue igual a cero, en las dosis de 0,20 y $0,13 \mathrm{mg}$ i.a/L del producto comercial para $T$. urticae y $P$. macropilis, respectivamente (Figura $1 \mathrm{~A}$ y B).

En cuanto a Organic Neem, la tasa instantánea de crecimiento poblacional fue igual a cero, en las dosis de 0,56 y $0,39 \mathrm{mg}$ i.a/L del producto comercial para T. urticae y P. macropilis, respectivamente (Figura $2 \mathrm{~A}$ y B).

Relativo a Natuneem, la tasa instantánea de crecimiento poblacional fue igual a cero en las dosis de 2,02 y $1,43 \mathrm{mg}$ i.a/L del producto comercial para T. urticae y P. macropilis, respectivamente (Figura $3 \mathrm{~A}$ y B).

La obtención del control de T.urticae, a bajas concentraciones, representa un gran avance en relación a los estudios que buscan el control de ese ácaro, considerado una de las más importantes plagas del cultivo de fresa.

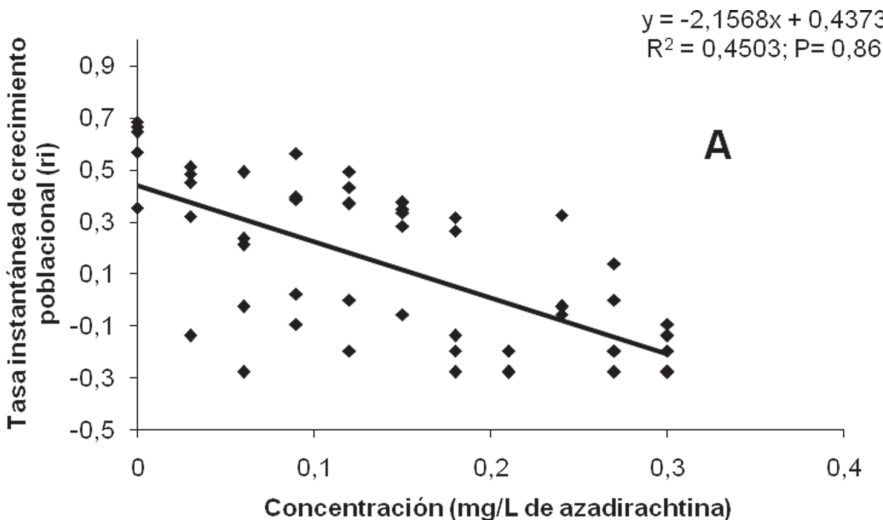

La aplicación de dosis subletales de los productos a base de nim en la población, se manifiesta en la reducción de la fecundidad, deterrencia, inviabilidad de formas inmaduras y repelencia, presentando, normalmente, selectividad para los enemigos naturales (Dimetry et al. 1993; Castiglioni et al. 2002; Schmutterer, 1997). Los productos a base de nim en las concentraciones manejadas, no afectaron al predador $P$. macropilis.

La aplicación de los productos a base de nim Neem Pro, Organic Neem y Natuneem representan una alternativa viable para el control del ácaro fitófago T. urticae en plantas de fresa, que se pueden integrar dentro de un programa de manejo integrado de dicha plaga, en concentraciones de azadirachtina, que posibiliten efectos negativos sobre el ácaro fitófago T. urticae, y que no afecte al ácaro predador P. macropilis.

Agradecimientos: A la Fundação de Amparo à Pesquisa do Estado de Minas Gerais (FAPEMIG), Brazil, por el financiamiento de la investigación. Conflictos de intereses:

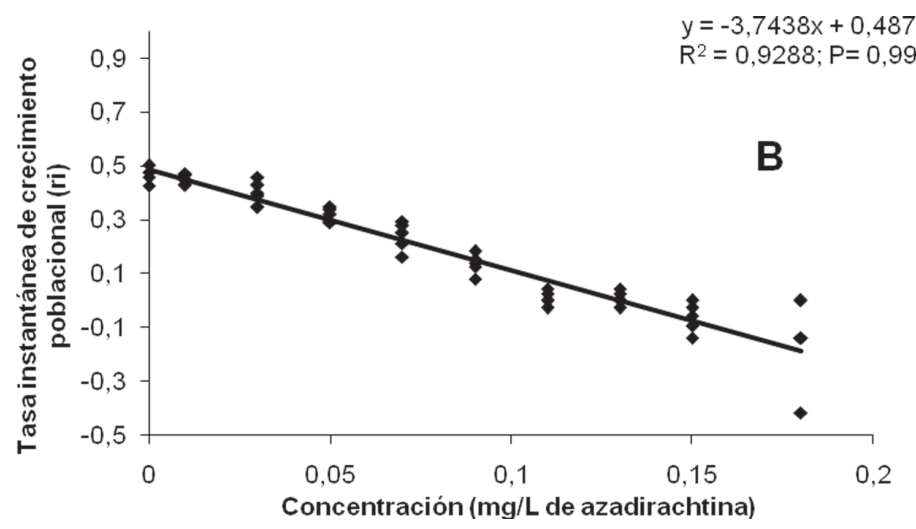

Figura 1. Tasa instantánea de crecimiento poblacional $\left(r_{i}\right)$ del ácaro A. T. urticae y, B. P. macropilis, en función de la aplicación de concentraciones del producto comercial Neem Pro. 

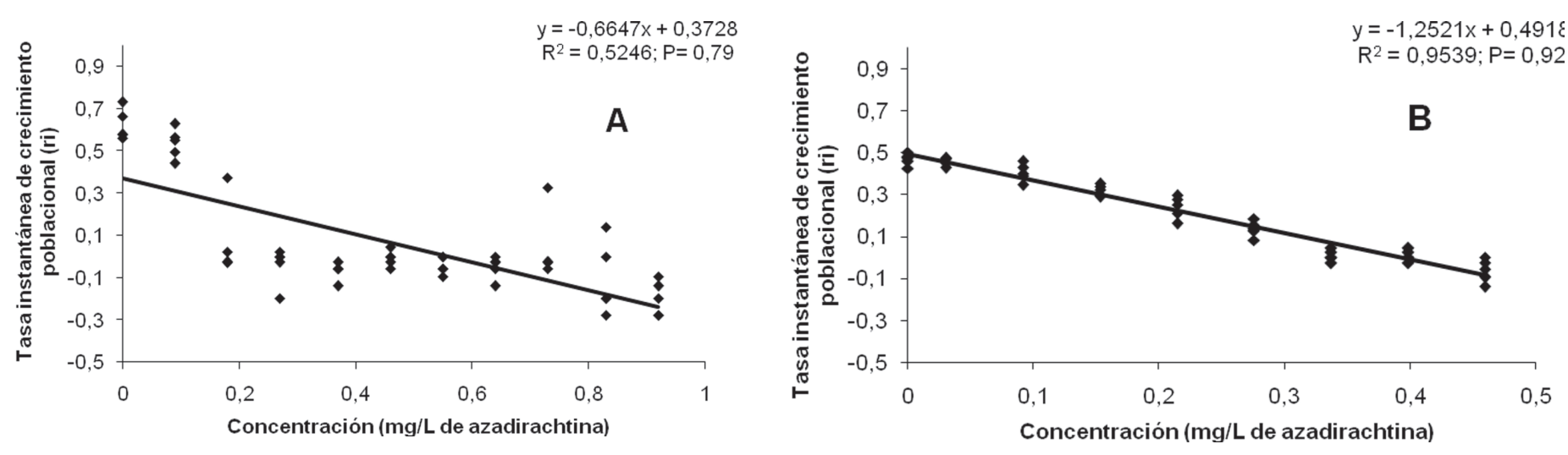

Figura 2. Tasa instantánea de crecimiento poblacional (ri) del ácaro A. T. urticae y B. P. macropilis, en función de la aplicación de concentraciones del producto comercial Organic Neem.
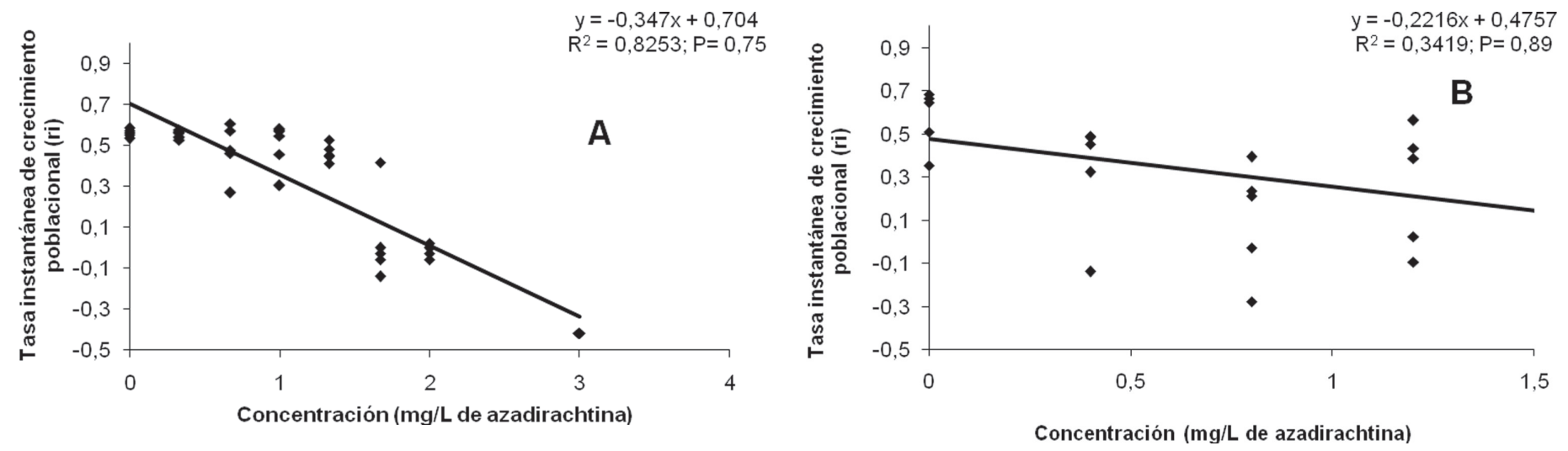

Figura 3. Tasa instantánea de crecimiento poblacional (ri) del ácaro A. T. urticae y B. P. macropilis, en función de la aplicación de concentraciones del producto comercial Natuneem.

El artículo fue preparado y revisado con la participación de todos los autores, quienes declaramos que no existe ningún conflicto de intereses que ponga en riesgo la validez de los resultados presentados.

\section{BIBLIOGRAFÍA}

1. BRITO, H.M.; GONDIM, M.G.C.; OLIVEIRA, J.V.; GOMES, C.A. 2006. Toxicidade de Natuneem sobre Tetranychus urticae Koch (Acari: Tetranychidae) e ácaros predadores da família Phytoseiidae. Ciência e Agrotecnología, Lavras 30(4):685-691.

2. CASTAGNOLI, M.; SIMONI, S.; GOGGIOLI, D. 2000. Attività biológica di sostanze vegetali nei confronti di Tetranychus urticae Koch (Acari: Tetranychidae) e del suo predatore Neoseiulus californicus (Mcgregor) (Acari: Phytoseiidae). Redia. 83:141-150.
3. CASTIGLIONI, E.; VENDRAMIM, J.D.; TAMAI, M.A. 2002. Evaluación del efecto tóxico de extractos acuosos y derivados de meliáceas sobre Tetranychus urticae (Koch) (Acari: Tetranychidae). Agrociência. 6:75-82.

4. CHIAVEGATO, L.G.; MISCHAN, M.M. 1981. Efeito do ácaro Tetranychus (T.) urticae (Koch, 1836) Boudreux; Dosse, 1963 (Acari: Tetranychidae) na produção no morangueiro (Fragaria spp) CV. Científica, Campinas 9:257-266.

5. DIMETRY, N.Z.; AMER, S.A.A.; REDA, A.S. 1993. Biological activity of two neem seed kernel extracts against the two-spotted spider mite Tetranychus urticae Koch. J. Appl. Entomol.116:308-312.

6. EL-GENGAIHI, S.; DIMETRY, N.Z.; AMER, S.A.A.; S.M. MOHAMED, S.M. 2000. Acaricidal activity of lipoidal 
matter of different plant extracts against the twospotted spider mite Tetranychus urticae Koch. Insect Science and its Application. 20(3):191-194.

7. FINNEY, D.J. 1971. Probit analysis. Cambridge: Cambridge University Press. 333p.

8. GARCÍA, I.P.; CHIAVEGATO, L.G. 1997. Respostas funcional e reprodutiva de Phytoseiulus macropilis (Banks, 1905) (Acari: Phytoseiidae) a diferentes densidades de ovos de Tetranychus urticae (Koch, 1836) (Acari: Tetranychidae). Científica 25:35-43.

9. GONÇALVES, M.E.C.; OLIVEIRA, J.V.; BARROS, R.; TORRES, J.B. 2001. Efeito de extratos vegetais sobre estágios imaturos e fêmeas adultas de Mononychellus tanajoa (Bondar) (Acari:Tetranychidae). Neotropical Entomology, Londrina. 30(2):305-309.

10. HERNÁNDEZ, C.R.; VENDRAMIM, J.D. 1996. Toxicidade de extratos aquosos de Meliácea em Spodoptera frugiperda (Lepidoptera: Noctuidae). Manejo Integrado de Pragas, Turrialba. 42:12-22.

11. MANSOUR, F.A.; ASCHER, K.R.S.; ABO-MOCH, F. 1993. Effects of Margosan-OTM, AzatinTM and RD9-Repelin on spiders, and on predacious and phytophagous mites. Phytoparasitica. 21(3):205-211.

12. MANSOUR, F.A.; ASCHER, K.R.S. 1983. Effects of neem (Azadirachta indica) seed kernel extracts from different solvents on the carmine spider mite, Tetranychus cinnabarinus. Phytoparasitica. 11:177-185.

13. MANSOUR, F.A.; ASCHER, K.R.S.; OMARI, N. 1987. Effects of neem (Azadirachta indica) seed kernel extracts from different solvents on the predacious mite Phytoseiulus persimilis and the phytophagous mite Tetranychus cinnabarinus. Phytoparasitica. $15: 125-130$.

14. MOMEN, F.M.; REDA, A.S.; AMER, S.A.A. 1997. Effect of Neem Azal-F on Tetranychus urticae and three predacious mites of the family Phytoseiidae. Acta Phytopathologica et Entomologica Hungarica 32:355-362

15. MOURÃO, S.A.; SILVA, J.C.T.; GUEDES, R.N.C.; VENZON, M.; JHAM, G.N.; OLIVEIRA, C.L.; ZANUNCIO, J.C. 2004. Seletividade de extratos de nim (Azadirachta indica A. Juss.) ao ácaro predador Iphiseiodes zuluagai (Denmark \& Muma) (Acari: Phytoseiidae). Neotropiocal Entomology. 33:613-617.
16. OLIVEIRA, H.; JANSSEN, M.; PALLINI, A.; VENZON, M.; FADINI, M.A.; DUARTE, M. 2007. A phytoseiid predator from the tropics as potencial biological control agent for the spider mite Tetranychus urticae Kock (Acari: Tetranychidae). Biological Control. 42: 105-109.

17. OVERMEER, W.P.J.; VANZON, A.Q. 1982. A standardized method for testing the side effect of pesticides on the predaceous mite, Amblyseius potentillae (Acari: Phytoseiidae). Entomophaga. 27:357-364.

18. POTTER, C. 1952. An improved laboratory apparatus for applying direct sprays and surface films, with data on the electrostatic charge on atomized spray fims. Annals of Applied Biology. 39:1-29.

19. POTENZA, M.R.; TAKEMATSU, A.P.; SIVIERI, A.P.; SATO, M.E.; PASSEROTTI, C.M. 1999a. Efeito acaricida de alguns extratos vegetais sobre Tetranychus urticae (Koch, 1836) (Acari: Tetranychidae) em laboratório. Arquivos do Instituto Biológico. 66:31-37.

20. POTENZA, M.R.; TAKEMATSU, A.P.; BENEDICTO, L.H. 1999b. Avaliação do controle de Tetranychus urticae (Koch, 1836) (Acari: Tetranychidae) através de extratos vegetais, em laboratório. Arquivos do Instituto Biologico. 66:91-97.

21. REIS, P.R.; CHIAVEGATO, L.G.; MORAES, G.J.; ALVES, E.B.; SOUSA, E.O. 1998. Seletividade de agroquímicos ao ácaro predador Iphiseiodes zuluagai Denmark E Muma (Acari: Phytoseiidae). Anais da Sociedade Entomológica do Brasil. 27:265-274.

22. REMBOLD, H. 1989. Azadirachtins: Their structure and mode of action. In: Arnason, J.T.; Philogene, B.J.R.; Morand, P. (eds.), Insecticides of plant origin. Washington, American Chemical Society. p.150-163.

23. SATO, M.E.; PASSEROTTI, C.M.; TAKEMATSU, A.P.; SOUZA Filho, M.F.; POTENZA, M.R.; SIVIERI, A.P. 2000. Resistência de Tetranychus urticae (Koch, 1836) a acaricidas, em pessegueiro (Prunus persica (L.) Batsch) em Paranapanema e Jundiaí-SP. Arquivos do Instituto Biológico. 67(1):117-123.

24. SATO, M.E.; SILVA, M. DA; GONÇALVEZ, L.R.; SOUZA FILHO, M.F. DE.; RAGA, A. 2002. Toxicidade diferencial de agroquímicos a Neoseiulus californicus (McGregor) (Acari: Phytoseiidae) e Tetranychus urticae Koch (Acari: Tetranychidae) a morangueiro. Neotropical Entomology, Londrina. 31(3):449-456. 
25. SCHMUTTERER, H. 1997. Insect growth-disrupting and fecundity reducing ingredients from the neem and chinaberry trees. In: E.D. Morgan, E.D.; Mandava, N.B. (eds.), CRC Handbook of natural pesticides. Florida, CRC series in naturally occurring pesticides. p.119-170.

26. SOTO, A.; VENZON, M.; OLIVEIRA, R.; OLIVEIRA, H.; PALLINI, A. 2010. Alternative control of Tetranychus evansi Baker E Pritchard (Acari: Tetranychidae) on tomato plants grown in greenhouses. Neotropical Entomology. 39(4):363-368.

27. SPOLLEN, K.M.; ISMAN, M.B. 1996. Acute and sublethal effects of a neem insecticide on the commercial biological control agents Phytoseiulus persimilis and Amblyseius cucumeris (Acari: Phytoseiidae) and Aphidoletes aphidimyza (Diptera: Cecidomyiidae). J. Econ. Entomol. 89:1379-1386.

28. STARK, J.D.; RANGUS, T. 1994. Lethal and sublethal effects of the neem insecticide, Margosan-O, on pea aphid. J. Pest Science. 41:155-160.

29. STARK, J.D.; VARGAS, R.I.; MESSING, R.H.; PURCELL, M. 1992. Effects of cyromazine and diazinon on three economically important Hawaiian Tephritidae fruit flies (Diptera: Tephritidae) and their endoparasitoids (Hymenoptera: Braconidae). J. Econ. Entomol. 85:1687-1694.
30. STARK, J.D.; BANKS, J.E. 2003. Population-level effects of pesticides and other toxicants on arthropods. Ann. Ver. Entomol. 48:505-519.

31. VENZON, M.; OLIVEIRA, H.; SOTO, A.; OLIVEIRA, R.M.; FREITAS, R.C.P.; LOPES, I.P.C. 2008. Potencial de produtos alternativos para o controle de pragas. In: Poltronieri, L.S.; Ishida, A.K.N. (eds.). Métodos alternativos de controle de insetos-praga, doenças e plantas daninhas. Belém, Embrapa Amazônia Oriental. p.263-287.

32. VIEGAS, JR., C. 2003. Terpenos com atividade inseticida: Uma alternativa para o controle químico de insetos. Química Nova. 26:390-400.

33. VINSON, S.B. 1974. Effect of an insect growth regulator on two parasitoids developing from treated tobacco budworm larvae. J. Econ. Entomol. 67:335-336.

34. WALTHALL, W.K.; STARK J.D. 1997. Comparison of two population-level ecotoxicological endpoints: the intrinsic $(\mathrm{rm})$ and instantaneous $(\mathrm{ri})$ rates of increase. Environmental Toxicology and Chemistry. 16:10681073.

Recibido: Febrero 9 de 2011

Aceptado: Abril 13 de 2011 